\title{
Extrapolation of serial stimulus patterns by rats
}

\author{
STEPHEN B. FOUNTAIN and STEWART H. HULSE \\ Johns Hopkins University, Baltimore, Maryland 21218
}

\begin{abstract}
This experiment determined if rats could extrapolate a familiar serial sequence of diminishing food quantities by accurately anticipating a novel quantity added to the end of the sequence. In 13 days of training, rats ran in a straight runway to obtain quantities of food pellets presented in sequential order. A strongly monotonic group received repetitions of a formally simple pattern of 14-7-3-1 pellets of food, while weakly monotonic and nonmonotonic groups received formally more complex 14-5-5-1 and 14-3-7-1 patterns, respectively. In subsequent transfer, a 0-pellet quantity was added to each pattern, thus extending pattern length to five elements. Results of the very first pattern repetition containing the added 0-pellet element indicated that rats in the strongly monotonic condition, but not in the others, anticipated the reduced quantity before actually experiencing it. This result supports a cognitive, rule-learning hypothesis for serial learning by rats.
\end{abstract}

An important assumption of cognitive models of serial pattern learning (Jones, 1974, 1976; Restle, 1970; Simon \& Kotovsky, 1963) is that subjects abstract formal pattern structure from a sequence of stimuli and encode a representation of the structure. The encoded representation is then used to recognize or regenerate the sequence, or to extend the serial pattern by supplying additional elements conforming to the sequence's underlying formal structure. Thus, when people learn a sequential pattern of numbers like 1234512345, it is presumed they encode a representation of the "increase" and "repeat" rules that characterize the formal structure of the pattern. The representation is then drawn upon, for example, to recall the pattern or to extend and extrapolate it by adding a 1 (and a 2345).

In studying serial pattern learning in rats, Hulse and Dorsky $(1977,1979)$ proposed that rats, like humans, are sensitive to the rule structures describing sequential patterns and encode in some as yet unknown way a representation of a pattern's formal structure. The present experiment extends their work by investigating rats' capacity to extrapolate a sequentially organized pattern of stimuli.

Hulse and Dorsky $(1977,1979)$ treated a set of food quantities as stimuli and constructed sequential patterns from them. Because these stimulus elements were drawn from an ordered stimulus

This research was supported by Biomedical Sciences Support Grant 5-507-RR07041-12 from the Department of Health, Education and Welfare to Johns Hopkins University. We thank Donna Karnoutsos, Karen Hansen, Ward Chow, and Bruce Lenes for their help in data collection, and Joan Krach for her help in manuscript preparation. Reprints may be obtained from Stewart H. Hulse, Department of Psychology, Johns Hopkins University, Baltimore, Maryland 21218. dimension, that of reward magnitude, they met the criteria for a stimulus "alphabet" (Jones, 1974) an important requirement for meaningful specification of formally defined serial patterns of stimuli. Sequences of differing formal complexity were constructed by arranging food quantities according to relatively simple or complex rule structures. A strongly monotonic sequence of 14-7-3-1-0 food pellets was the formally simplest sequence used, requiring a single "less than" rule to relate the magnitude of each successive pattern element. A weakly monotonic 14-5-5-1-0 and a nonmonotonic 14-1-3-7-0 pattern were progressively more complex, requiring combinations of "less than," "greater than," or "equal" rules. Rats learned to track the sequential patterns by running fast or slowly in anticipation of large or small quantities when the quantities were presented one after the other on consecutive runs in a runway. By this measure, Hulse and Dorsky (1977) found that the formally simple monotonic pattern was learned most rapidly and to the most proficient level of tracking performance followed by the weakly monotonic and nonmonotonic patterns.

Hulse and Dorsky's results are analogous to previous results of Restle and Brown (1970) which showed that humans easily learn patterns based on formally simple sequential structures such as "runs" or "trills" that can be described formally by the repeated application of a single rule, for example, 12345 or 12121 . Restle and Brown (1970) also found that human subjects have a predilection to extrapolate such formally simple patterns, but are less prone to extrapolate formally complex patterns. This is consistent with the idea that the subject's representation of the formally simple pattern readily yields the next logical stimulus in the series, while the representation of a 
formally complex pattern does not obviously do so.

In the experiment we report now, we tested rats' ability to extrapolate serial patterns of food quantities when we systematically varied the formal complexity of the pattern the rats were to extrapolate. During an initial training phase, three patterns of varying formal complexity were presented repeatedly to different groups of rats. This served to expose the rats to the formally defined stimulus structures they were to extrapolate later. A strongly monotonic pattern of 14-7-3-1 food pellets, described by a single "less than" rule, was the simplest used. Two more complex patterns were a weakly monotonic pattern of 14-5-5-1 food pellets and a nonmonotonic pattern of 14-3-7-1 food pellets. The latter required combinations of "less than," "greater than," and "equal" rules for a complete formal description. During a subsequent transfer phase, the rats responded to a novel 0-pellet quantity added to the end of the sequence. The rule-learning hypothesis predicts that rats exposed to the formally simple, strongly monotonic sequence in the first phase should extrapolate the sequence to anticipate a further reduction in food quantity on the very first pattern in which the reduction occurs, that is, prior to actually experiencing the 0-pellet quantity. Anticipation should be reflected in slower running times to the 0 -pellet quantity. Accurate anticipation of a reduced quantity should be progressively more difficult, if possible at all, in the formally more complex patterns because their formal structure implies a small or 0-pellet quantity less clearly. Accordingly, there should be proportionately little change in response times in anticipation of the new added element.

\section{METHOD}

\section{Subjects}

The subjects were 30 Sprague-Dawley rats obtained from ARS/ Sprague-Dawley, Madison, Wisconsin, at 90 to 100 days of age. The rats were 100 to 120 days of age at the beginning of the experiment. The animals were housed in individual cages and were maintained at $85 \%$ of ad-lib weight throughout the experiment.

\section{Apparatus}

The apparatus was the straight enclosed runway described by Hulse and Dorsky (1977). The runway was $92 \mathrm{~cm}$ long, with a $30-\mathrm{cm}$ startbox and a $30-\mathrm{cm}$ goal area separated from the runway by guillotine doors. The goal area included an $11-\mathrm{cm}$ niche at a right angle to the runway. The niche contained a food cup consisting of an inverted jar lid $(5 \mathrm{~cm}$ in diameter) fastened to the floor. The inside width throughout was $10 \mathrm{~cm}$, and the inside height was $11 \mathrm{~cm}$. The start and goal areas were covered with pieces of hinged Plexiglas, while the runway proper was covered with hardware cloth. The apparatus was painted flat black except for the start area, which was natural wood color. A timer started when the startbox door was raised and stopped when the rat activated a contact relay by bridging a gap between two metal plates that was located $20 \mathrm{~cm}$ into the goal area. Food reinforcement consisted of an appropriate number of $45-\mathrm{mg}$ Noyes food pellets.

\section{Procedure}

All rats received the same treatment for 1 week prior to the beginning of the experiment. The rats were tamed by handling approximately $10 \mathrm{~min}$ each day and were reduced to $85 \%$ of normal ad-lib weight during this period. Exploration of the apparatus was allowed on the 2 days prior to experimental testing. On the first day of exploration, both guillotine doors of the apparatus were open and individual animals were allowed to run through the apparatus for $10 \mathrm{~min}$, obtaining 4 food pellets scattered at random and 1 food pellet located in the food cup. On the second day, the rats were placed in the startbox and then released when the startbox door was raised. During a 5-min exploration period, they could eat 1 food pellet located in the food cup. This procedure was repeated once, with the rat returned to its home cage for a 10- to 15 -min interval between exploration periods.

On the day following exploration, the training phase of the experiment began. The rats were assigned randomly to three conditions, 10 rats per condition. The strongly monotonic (S) group was presented four-element pattern repetitions of 14-7-3-1 pellets of food. The weakly monotonic (W) group received pattern repetitions of 14-5-5-1 pellets of food. Finally, the nonmonotonic $(N)$ group received pattern repetitions of 14-3-7-1 pellets of food.

The training phase of the experiment lasted for 13 days. The rats received two four-element pattern repetitions on Day 1 of training, and four pattern repetitions per day for Days 2 through 13. Each day rats were tested in squads of three and four animals. A pattern repetition began when the first rat was placed in the startbox of the runway. A run to the appropriate quantity began when the startbox and goalbox were opened to allow the animal to proceed through the runway. The run ended when the rat entered the goal area and stopped the timer or when a nonresponse criterion of $60 \mathrm{sec}$ had elapsed without the animal's entering the goal area. If the rat entered the goal area, it was confined there until all food was consumed. The rat was then placed in a holding cage for 10 to $15 \mathrm{sec}$ while the goal was baited and the run time was recorded. If the nonresponse time elapsed, the rat was removed immediately from the runway and placed in the holding cage to await the next run. Runs continued in this fashion until all four runs of the pattern repetition were completed. The rat was returned to its home cage for a period of 10 to 15 min until the next pattern repetition began. During this interval, the other rats in the squad were tested. Water was freely available in the home cages at all times.

The transfer phase of the experiment began on Day 14. All rats received the first pattern repetition of the day exactly as it had been presented during the previous training phase. On the second pattern repetition of the day, however, the procedure was modified so that each pattern consisted of five runs. The rats in each group received the first four quantities as they had experienced them in previous training. Following the usual 10- to $15-\mathrm{sec}$ interval, they ran a fifth run in which they found no food in the goal area, that is, a 0-pellet quantity. Confinement in the goalbox for 0 pellets was for $15 \mathrm{sec}$. As in the previous training phase, a nonresponse criterion of $60 \mathrm{sec}$ was in effect. The transfer phase continued through Day 15 , that is, rats received one fourelement pattern and three five-element patterns on Day 14 and four five-element patterns on Day 15.

\section{RESULTS}

Figure 1 shows the results of the first pattern repetition in which the 0 -pellet quantity was presented, that is, the extrapolation pattern repetition of Day 14 . For each group, mean running times are shown for the quantities in the order in which they were presented. The results show that rats correctly anticipated the very first presentation of the 0-pellet quantity, slowing their running times appropriately. $\mathrm{W}$ rats and $\mathrm{N}$ rats failed to slow very much in anticipation of the 0-pellet quantity. The running times 


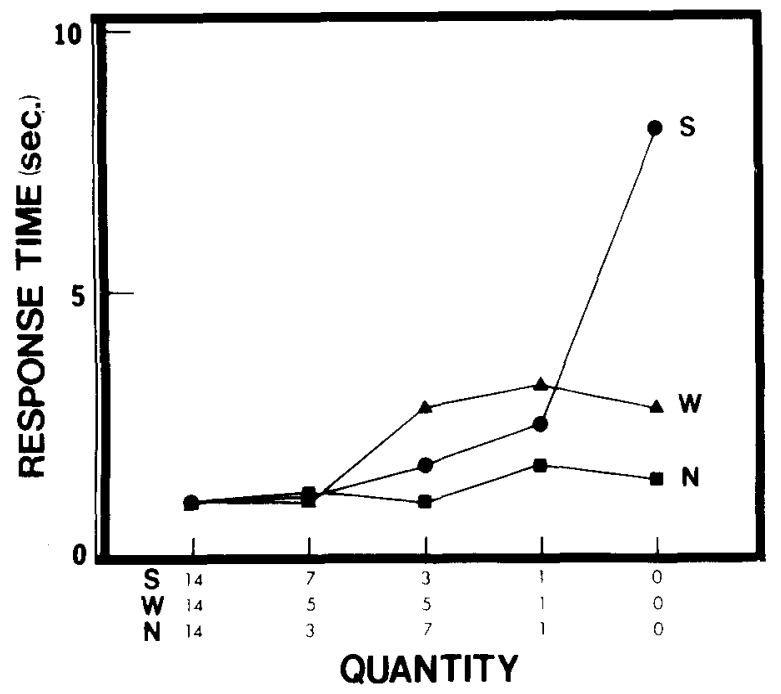

Figure 1. Mean running times on the first pattern repetition of the transfer phase for strongly monotonic (S), weakly monotonic (W), and nonmonotonic $(N)$ conditions. The $S$ pattern was 14-7-3-1-0 food pellets, the $W$ pattern was 14-5-5-1-0, and the $N$ pattern was 14-3-7-1-1 pellets of food.

to 0 pellets were ordered according to formal complexity of the patterns, that is, the $\mathrm{W}$ condition led to slower running than the $\mathbf{N}$ condition. However, the differences were not large. Furthermore, the running times to 0 pellets were not appreciably slower in the $\mathrm{W}$ condition than to earlier quantities in that sequence.

An analysis of variance on these data indicated a significant main effect for response times to the different quantities $[F(4,27)=3.84, p<.01]$ and a reliable Group by Quantities interaction $[F(8,108)=2.26$, $\mathrm{p}<.05]$. A subsequent Newman-Keuls test indicated that the $S$ animals ran reliably more slowly to the 0 pellet quantity than to any of the other quantities within the strongly monotonic pattern. They also ran significantly more slowly to the 0-pellet quantity than animals in the $\mathrm{W}$ and $\mathrm{N}$ groups ran to any of the quantities in their respective patterns $(p<.01)$. Other differences in running times both within and across conditions were not reliable by the NewmanKeuls test $(p>.05)$.

An analysis of variance on the mean running time data for all seven transfer repetitions indicated significant main effects for Groups $[F(2,27)=14.52$, $\mathrm{p}<.01]$ and Quantities $[\mathrm{F}(4,108)=24.53, \mathrm{p}<.01]$, and a reliable Group by Quantities interaction $[F(8,108)=13.63, p<.01]$. None of the effects involving Repetitions was significant $(p>.05)$. Inspection of the data revealed that results for the entire transfer phase mirrored the results seen for the very first pattern repetition of the transfer phase. Subsequent Newman-Keuls tests showed that rats in the $S$ condition continued to run more slowly to the 0 - pellet quantity than to any other quantity within the $S$ pattern $(p<.01)$, while rats in both $W$ and $N$ conditions never slowed reliably to any of the smaller quantities they received $(p>.05)$.

A control analysis was performed to compare responding in the training phase with responding in the first pattern repetition of the transfer phase. Because no changes in conditions occurred until a 0 -pellet quantity was appended to the second pattern repetition of Day 14, results for the first pattern repetition and for the first four runs of the second pattern repetition of Day 14 should be similar. Response times for these data were subjected to an analysis of variance. The analysis indicated reliable main effects for Repetitions $[F(1,27)=4.67, p<.05]$ and for Quantities $[F(3,81)=4.44, p<.01]$, but no significant effect for Groups or any of the interactions ( $p s>.05$ ). Rats in all groups slowed running times to the 1-pellet quantity. This effect was small (viz., mean response times to the 14- and 1-pellet quantities differed by $.8 \mathrm{sec}$ ) and, in spite of the significant overall effect for Quantities, a Newman-Keuls test comparing running times for the four quantities found no reliable differences $(p>.05)$. The significant Repetitions effect was due to slightly slower responding to all quantities on the second pattern repetition of the day (mean response time for the first repetition was $1.3 \mathrm{sec}$ compared with $1.6 \mathrm{sec}$ for the second repetition). Despite the significant Repetitions effect of the analysis of variance, no significant differences among the relevant means were obtained with a Newman-Keuls test $(p>.05)$.

In the same vein, a control analysis was performed on daily means of the training phase prior to the day of transfer. Responding throughout the training phase was similar to responding to the first four quantities of the first transfer pattern repetition. An analysis of variance on these data indicated reliable main effects for Days $[F(12,234)=18.18, p<.01]$ and Quantities $[F(3,81)=20.81, p<.01]$, but no reliable effects for Groups or any of the interactions $(p>.05)$. The Days effect reflected general reductions in running time over the first days of the experiment. The Quantities effect reflected slower running to the 1-pellet quantity than to the other quantities. However, these latter differences were not reliable by a Newman-Keuls test $(p>.05)$.

\section{DISCUSSION}

The animals trained with a strongly monotonic pattern ran significantly more slowly on the initial fifth run in anticipation of the first presentation of the 0 -pellet quantity than did the animals in either the weakly monotonic or the nonmonotonic groups. That is, the rats in the $S$ group extrapolated the pattern. How can these results be explained most effectively, given that the animals' performance in all 
three conditions was based on no past experience with a fifth run to a 0 -pellet quantity?

\section{Rule Learning}

The data support predictions about pattern extrapolation that follow a cognitive model of serial pattern learning. That model postulates that organisms encode a representation of the formal rules used to construct a pattern, and that they can then use that representation to make predictions about novel elements added to the pattern. Also, given that formally simple patterns are easier to learn and, presumably, to encode than formally complex patterns (Hulse, 1978; Hulse \& Dorsky, 1977, 1979; Restle \& Brown, 1970), accurate extrapolation should be directly correlated with pattern complexity. The data fit both propositions. Rats in the formally simple monotonic condition extrapolated the pattern. While rats in the formally more complex conditions did not do so reliably, there was a trend in the correct direction in the weakly monotonic condition.

We note in passing that our data are in line with some observations of Jensen and Rey $(1968,1969)$. They ran rats in a runway to a goalbox that always contained a single pellet in a food cup. Above the cup was a transparent Plexiglas tube. Under a diminishing-food-supply (DFS) condition, the tube contained a supply of food pellets that diminished one by one from 15 to 0 pellets as 16 trials of training ran their course. The rats first increased their running speeds during early training trials, but began to run more and more slowly as trials progressed. This was true as compared with both their own early performance and the performance of several control groups in which rats were also rewarded with one food pellet per trial without the opportunity to observe the food supply diminish. By the time the tube was empty, running times in the DFS condition had returned virtually to an operant level.

We do not claim that the rats of Jensen and Rey had necessarily encoded some relation such as the "less than" rule we propose for our animals because, among other things, Jensen and Rey's rats had only one opportunity to observe their food supply decrease over trials. We believe, however, that Jensen and Rey's data lend credence to the proposition that rats can anticipate the implied fate of a serially structured sequence of food stimuli. Our data suggest that the process can, in addition, become internalized and representational. The form and properties of that representation remain, of course, to be determined.

\section{Other Possibilities}

In earlier work, Hulse and Dorsky $(1977,1979)$ obtained several sources of evidence directly against a theory of serial pattern learning, stressing, for example, pairwise stimulus associations among sequences of food quantities. For the present case, it is not clear which, if any, associative mechanisms would apply when an organism is asked to respond in anticipation of a never-before-experienced stimulus. One possible approach would be based on stimulus generalization decrement. Because all animals were trained for 13 days with patterns composed of four quantities, the five-element transfer pattern was novel. For this reason, one might expect a slowing of running times on the extra run to 0 pellets regardless of either the quantity to be received on the new run or the previous training sequence. Because the results indicate that only one of the three conditions, the strongly monotonic condition, generated slow running on the extra run, stimulus generalization decrement is not an adequate explanation of the effect.

While the foregoing is true, we are loathe to erect stimulus generalization decrement or any other process as a straw man generally representative of any putative mechanism that might be ascribed to our data. For now we claim only that a cognitive analysis suggested an original and useful experiment and appears to offer a highly parsimonious explanation of the behavior we consequently observed.

\section{REFERENCES}

Hulse, S. H. Cognitive structure and serial pattern learning by animals. In S. H. Hulse, H. Fowler, \& W. K. Honig (Eds.), Cognitive processes in animal behavior. Hillsdale, N.J: Erlbaum, 1978.

Hulse, S. H., \& Dorsky, N. P. Structural complexity as a determinant of serial pattern learning. Learning and Motivation, $1977,8,488-506$.

Hulse, S. H., \& Dorsky, N. P. Serial pattern learning by rats: Transfer of a formally defined stimulus relationship and the significance of nonreinforcement. Animal Learning and Motivation, 1979, 7, 211-220.

JENSEN, G. D., \& REY, R. P. Runway performance under "horn of plenty" conditions versus gradual diminution of reward supply. Journal of Experimental Psychology, 1968, 76, 7-11.

Jensen, G. D., \& REY, R. P. "Horn of plenty" conditions versus gradual diminution of reward supply with extended training. Journal of Experimental Psychology, 1969, 80, 190-191.

Jones, M. R. Cognitive representations of serial patterns. In B. Kantowitz (Ed.), Human information processing: Tutorials in performance and cognition. Hillsdale, N.J: Erlbaum, 1974.

JoNES, M. R. Levels of structure in the reconstruction of temporal and spatial serial patterns. Journal of Experimental Psychology: Human Learning and Memory, 1976, 2, 475-488.

Restle, F. Theory of serial pattern learning: Structural trees. Psychological Review, 1970, 77, 481-495.

Restle, F., \& BRown, E. R. Serial pattern learning. Journal of Experimental Psychology, 1970, 83, 120-125.

Simon, H. A., \& Kotovsky, K. Human acquisition of concepts for sequential patterns. Psychological Review, 1963, 70, 534-546.

(Received for publication October 28, 1980; accepted January 27, 1981.) 